\title{
Comment on Rosenberg J, et al.: "Deep Neuromuscular Blockade Improves Laparoscopic Surgical Conditions: A Randomized, Controlled Study"
}

\author{
Michiel C. Warlé · Albert Dahan
}

Received: April 1, 2017 / Published online: June 19, 2017

(C) The Author(s) 2017. This article is an open access publication

Rosenberg et al. presented a unique randomized clinical trial in which the use of deep versus moderate neuromuscular blockade during low or standard pressure laparoscopic cholecystectomy was systematically studied [1]. In our view three important issues should be addressed regarding this work.

First, with 11 participating centers the external validity and generalizability of the results are probably very good. However, in many cases there is a trade-off between external and internal validity. As the number of inclusions per center is relatively low it may have been difficult to control for differences between centers which may have introduced a certain degree of imprecision in outcome measures. As the sample size calculation did not control for multiple centers, it probably would have been better to perform this study with a limited number of centers.

Second, the authors used an 11-point Likert scale for the quality of surgical conditions rated by the first operator directly after surgery. This approach is different as compared other studies

M. C. Warlé $(\bowtie)$

Department of Vascular and Transplant Surgery,

Radboud University Medical Center, Nijmegen,

The Netherlands

e-mail: Michiel.Warle@radboudumc.nl

A. Dahan

Department of Anesthesiology, Leiden University

Medical Center, Leiden, The Netherlands investigating surgical conditions during laparoscopic surgery. To date the best validated method to rate the quality of the surgical field during laparoscopy is the Leiden-Surgical Rating Scale (Leiden-SRS) [2]. The L-SRS uses a 5-point Likert scale ranging from 1 (extremely poor) to 5 (excellent). Also the three most important factors influencing the quality of the surgical field are incorporated in the L-SRS including visibility of critical structures, working space, and muscle contractions. It is also important to note that the L-SRS is developed for use during surgery, e.g., for titration towards the lowest possible insufflation pressure. In the study by Rosenberg et al., surgical conditions were not systematically rated during surgery; however, the intra-abdominal pressure and/or degree of neuromuscular blockade was increased when conditions were "unacceptable". As a clear definition of "unacceptable" conditions is not described, this may have been a source of heterogeneity. Therefore we advocate the use of the Leiden-SRS for future studies. Also this might help to find explanations for differences between studies.

Finally, the authors conclude that the use of low pressure did not reduce (referred) pain scores. They refer to a systematic review and meta-analyses by Gurusamy et al. to support this conclusion [3]. However, in this review Gurusamy et al. stated that postoperative pain scores are unvalidated surrogate outcomes for 
pain after laparoscopic cholecystectomy. In our view this does not necessarily confirm the conclusion that the use of low pressure pneumoperitoneum does not reduce postoperative (referred) pain scores. Moreover, a systematic review and meta-analysis performed by our group on the use of low pressure during laparoscopic surgery showed that low pressure is associated with lower (referred) pain scores supported by moderate quality of evidence [4].

\section{ACKNOWLEDGEMENTS}

No funding or sponsorship was received for the publication of this article or the open access fee. All named authors meet the International Committee of Medical Journal Editors (ICMJE) criteria for authorship for this manuscript, take responsibility for the integrity of the work as a whole, and have given final approval for the version to be published.

Disclosures. Michiel C. Warlé received unrestricted grants for investigator-driven studies from Merck. Albert Dahan received unrestricted grants for investigator-driven studies from Merck.

Compliance with Ethics Guidelines. This article is based on previously conducted studies and does not involve any new studies of human or animal subjects performed by any of the authors.
Open Access. This article is distributed under the terms of the Creative Commons Attribution-NonCommercial 4.0 International License (http://creativecommons.org/licenses/ by-nc/4.0/), which permits any noncommercial use, distribution, and reproduction in any medium, provided you give appropriate credit to the original author(s) and the source, provide a link to the Creative Commons license, and indicate if changes were made.

\section{REFERENCES}

1. Rosenberg J, Herring WJ, Blobner M, et al. Deep neuromuscular blockade improves laparoscopic surgical conditions: a randomized controlled study. Adv Ther. 2017;34(4):925-36.

2. Martini CH, Boon M, Bevers RF, Aarts LP, Dahan A. Evaluation of surgical conditions during laparoscopic surgery in patients with moderate versus deep neuromuscular block. Br J Anaesth. 2014;112(3):498-505. doi:10.1093/bja/aet377.

3. Gurusamy KS, Vaughan J, Davidson BR. Low pressure versus standard pressure pneumoperitoneum in laparoscopic cholecystectomy. Cochrane Database Syst Rev. 2014. doi:10.1002/14651858.CD006930. pub3.

4. Özdemir-van Brunschot DM, van Laarhoven KC, Scheffer GJ, Pouwels S, Wever KE, Warlé MC. What is the evidence for the use of low-pressure pneumoperitoneum? A systematic review. Surg Endosc. 2016;30(5):2049-65. 